\title{
El impacto de los espacios formativos acerca de las competencias sociales y laborales en la carrera de medicina veterinaria
}

\author{
Fascendini $\mathrm{PY}^{1^{*}}$, Guaita $\mathrm{ML}^{1}$, Peralta $\mathrm{J}^{1}$, De Gennaro $\mathrm{M}^{1}$ \\ ${ }^{1}$ Facultad de Ciencias Veterinarias, Universidad Nacional del Litoral, Argentina. \\ * Correspondencia: Paola Fascendini, FCV-UNL, RP Kreder 2805 (3080) \\ Esperanza, Santa Fe, Argentina. \\ E-mail: rivas.francisco@conicet.gov.ar
}

Recibido: 28 Febrero 2019. Aceptado: 19 Julio 2019. Disponible en línea: 9 Octubre 2019

Editor: P. Beldomenico

\begin{abstract}
RESUMEN. El presente trabajo da cuenta de una propuesta de espacios formativos destinados a la adquisición de habilidades sociales en los estudiantes próximos a finalizar la carrera de Medicina Veterinaria de la Facultad de Ciencias Veterinarias de la Universidad Nacional del Litoral, Argentina. Dichos espacios, brindados desde el Servicio de Orientación Educativa, se organizaron en cuatro talleres inspirados en la teoría de las habilidades sociales y laborales. Los cuatro ejes principales fueron autoconocimiento, inserción laboral, habilidades sociales y competencias laborales. Los cuatros ejes principales fueron: autoconocimiento, inserción laboral, habilidades sociales y competencias laborales. El valor pedagógico de la propuesta se evaluó a través de la aplicación de encuestas de opinión y valoraciones de frases significativas. La metodología de abordaje se caracterizó por ser cualitativa, consistente en una estrategia de intervención dentro del ámbito educativo. Algunos resultados preliminares dan cuenta de la necesidad de articular talleres de habilidades sociales a la estructura curricular de la formación universitaria. Los protagonistas de este proceso enfatizan la significatividad que aportan estos espacios como oportunidades válidas para su formación, a la vez que se transforman en instancias de aprendizaje compartido.
\end{abstract}

SUMMARY. The impact of training spaces on social and labor competences in the career of veterinary medicine. The present work accounts for a proposal of training spaces aimed at the acquisition of social skills in students close to finishing the career of Veterinary Medicine of the Faculty of Veterinary Sciences of Universidad Nacional del Litoral, Argentina. These spaces, provided by the Educational Guidance Service, were organized in four workshops inspired by the theory of social and work skills. The four main axes were self-knowledge, labor insertion, social skills and labor competencies. The four main axes were: self-knowledge, labor insertion, social skills and labor competencies. The pedagogical value of the proposal was evaluated through the application of opinion surveys and judgments of significant sentences. The approach methodology was characterized as being qualitative, consisting of an intervention strategy within the educational environment. Some preliminary results show the need to articulate social skills workshops to the curricular structure of university education. The protagonists of this process emphasize the significance that these spaces provide as valid opportunities for their formation, while they are transformed into instances of shared learning.

Palabras clave: espacios formativos, competencias sociales y laborales, educación veterinaria

Keywords: training spaces, social and labor competences, veterinary education

\section{Introducción}

Según transcurren los años y ante el vertiginoso desarrollo tecnológico, las incumbencias profesionales y los requerimientos laborales van modificándose, haciéndose necesario dotar a los futuros egresantes de habilidades sociales que les posibiliten una inserción exitosa en los diversos contextos laborales. Hablar de habilidades implica vincularse con el dominio de conceptos, destrezas, actitudes resultando que una persona competente es aquella que tiene el conocimiento declarativo (la información y conceptos), es decir, sabe lo que hace, por qué lo hace y conoce el objeto sobre el que actúa. Ser competente implica, tener la capacidad de ejecución, es decir el conocimiento procesal o las destrezas intelectuales y psicomotoras para en efecto llevar a cabo la ejecución sobre el objeto (Perrenoud, 1997). Las competencias humanas permiten generar aprendizajes comprensivos resultado de la totalidad de experiencias educativas formales e informales, manifestada en multiplicidad de situaciones y escenarios como parte del comportamiento, son capacidades generales que acompañan el desarrollo a partir de lo cual la persona construye una capacidad para llevar a cabo multiplicidad de tareas en una forma que es considerada como eficiente o apropiada. Las competencias son un fenómeno complejo ya que intervienen una serie de elementos que se inter- 
relacionan ya sea en la relación dialéctica del sujeto (conocimiento) y el entorno e implican un acto inicial de reconocimiento de la situación planteada sea real o ficticia (Wagensberg, 1998). Así, ser competente consiste en realizar una actuación exitosa eligiendo la respuesta adecuada - entre una gama de posibles respuestas - ante una cuestión que nos plantea el contexto. El individuo será de este modo el constructor de sus competencias (Le Boterf, 2000), ya que éstas combinan esa pericia con el comportamiento social. Por ejemplo, la aptitud para trabajar en equipo, la capacidad de iniciativa y la de asumir riesgos. Las mismas no sólo se aprenden en ámbitos educativos sino que también resultan del empeño y desempeño que por sus cualidades innatas o adquiridas subjetivas, posibilita combinar conocimientos teóricos y prácticos que lo llevan a construir la capacidad de comunicarse, de trabajar con los demás, de afrontar y solucionar conflictos. Para el progreso de una habilidad se requiere no sólo de procesos cognitivos avanzados sino también del ejercicio e intervención de actitudes del sujeto como factor de relevancia, siendo que conducen a establecer prioridades que pueden estimular o desestimular el interés por el desarrollo de determinadas habilidades. Ésta no se desarrolla para un momento o acción determinada, sino que se convierte en una cualidad, en una forma de respuesta aplicable a múltiples situaciones que comparten esencialmente la misma naturaleza, de allí que se hable de que las habilidades desarrolladas por un individuo configuran una forma peculiar de resolver tareas o problemas en diversas áreas. En los diferentes ámbitos laborales donde el médico veterinario se desempeña y considerando la formación que recibe en su carrera, se observan durante los años de cursado y a lo largo de cada uno de los espacios formativos, que se ofrece la capacitación necesaria para la adquisición de las competencias cognitivas y técnicas que harán posible el ejercicio de la medicina veterinaria, surgiendo, sin embargo, en las voces de los docentes y estudiantes, la necesidad de encontrar espacios para el aprendizaje y la resignificación de aquellas habilidades que hacen posible a los estudiantes su vinculación con el mundo del trabajo.

¿Qué pasos debe seguir un estudiante que egresa? ¿Cuáles son los requisitos para la matriculación? ¿Cómo se confecciona un Curriculum Vitae? ¿Qué aspectos se deben tener en cuenta en una entrevista laboral? ¿Qué competencias sociales debe tener un Médico Veterinario? ¿Cómo ofrecer a los estudiantes la posibilidad de valorar sus capacidades y su formación?, estos y otros muchos interrogantes, dieron origen a la planificación de un espacio en formato taller, desde donde se pretendió responder a esta demanda, articulado en ésta oportunidad con uno de los docentes de la guardia, quien detecta en forma colaborativa la necesidad de ir incursionando y forjando espacios constructivos de formación, por encontrarse un tanto carente. Por lo que los talleres fueron transversalizados en los tiempos del cursado de la guardia de pequeños y grandes animales (orientaciones que ofrece nuestra facultad) con el fin de ir instrumentando instancias previas a la futura inserción laboral, analizando variables diversas, en pos de la construcción de las propias herramientas necesarias para responder a las demandas del contexto actual.

\section{Presentación y desarrollo de la propuesta}

La mayoría de nuestros aprendizajes se producen en contextos de interacción social y son modulados por esta interacción. Ello es aún más evidente cuando hablamos de aprender habilidades sociales, no sólo por el carácter social del resultado de lo aprendido, sino también por los procesos implicados los que son necesariamente sociales y culturales (Pozo, 2008). Teniendo en cuenta dicha caracterización se requirió instrumentar un proceso de abordaje cualitativo buscando introducir cambios y mejorando la manera de concebir la práctica educativa, siendo todos los escenarios y personas dignas de estudio (Taylor y Bodgan, 2000). El enfoque cualitativo permite dar voz a los participantes atribuyendo un valor primordial a los significados sociales que las personas asignan al mundo que las rodea. Los actores sociales asignan significado a situaciones, a otras personas, a las cosas y a sí mismos a través de un proceso de interpretación de las necesidades. Esta intervención fue enriquecida por la mirada docente, quien en su análisis permitió suscitar espacios de transversalidad en la construcción de habilidades sociales, mediatizados a través de cuatro talleres denominados "insertándonos en el mundo laboral: competencias sociales y laborales", con una frecuencia semanal, de tres horas reloj por cada taller, en el mes de noviembre del ciclo académico 2018. Asistieron al taller 40 futuros egresantes de la carrera, de características heterogéneas, procedentes de diversos puntos del país.

Los talleres giraron en torno a cuatro ejes primordiales consistentes en el autoconocimiento, exploración del contexto laboral, habilidades sociales y competencias laborales, siendo las habilidades sociales priorizadas: el conocimiento de sí mismo, empatía, comunicación efectiva o asertiva, relaciones interpersonales, manejo de tensiones y estrés.

Para poder evaluar cada uno de los talleres se dispuso de la confección y aplicación de encuestas de opiniones, valoraciones en frases significativas, aportes personales que permitieran dar cuenta del proceso llevado a cabo y primoridalmente del impacto de estos espacios formativos.

\section{Desarrollo del primer taller: autoconocimiento}

El primer taller, centrado en el autoconocimiento promovió la búsqueda de las competencias vinculadas a la adaptabilidad y flexibilidad laboral necesaria para la detección de capacidades personales y localización de obstáculos que pudieran interferir en el proceso de ingreso al mundo laboral. Los conocimientos especia- 
lizados son importantes para la gestión, sobre todo en un mundo en el que imperan la alta competitividad, la necesidad de formación continua, la exigencia de marcar distancias con los otros, no obstante, el aspecto humano y la gestión del talento son en este caso el centro de toda acción. ¿Para qué sirve tener los mejores conocimientos sobre un área concreta, si en el plano práctico se es incapaz de gestionar un grupo? Para tal fin se administraron técnicas (Gismero González, 2000) que permitieron indagar acerca de los temores e inseguridades personales, actuando de termómetro de las dificultades pero a la vez promoviendo el desarrollo de la resiliencia, la proactividad y la asertividad como claves competentes en el proceso de formación integral. Una de las técnicas auto aplicables denominada EHS (Escala de Habilidades sociales), la que se encuentra compuesta por 33 ítems, explora la conducta habitual del sujeto en situaciones concretas y valora hasta qué punto las habilidades sociales modulan estas actitudes. El evaluador dispone de un índice Global del nivel de habilidades sociales o aserción de los sujetos analizados y además detecta, de forma individualizada, cuáles son las áreas más problemáticas a la hora de tener que comportarse de manera asertiva. Para ello, se organizan puntuaciones en seis factores o sub escalas: autoexpresión en situaciones sociales, defensa de los propios derechos como consumidor, expresión de enfado o disconformidad, decir no y cortar interacciones, hacer peticiones e iniciar interacciones positivas con el sexo opuesto. Cada una de estas variables permitió un análisis discriminado de las posibilidades que cada uno tiene en cuanto a puntos fuertes y de los aspectos que debe reencauzar para acceder a la conquista de competencias sociales y laborales. Luego se realizó una puesta en común de cada perfil propiciando instancias de análisis, comparación de los aspectos alcanzados y llevando a cabo un proceso de introspección necesaria para la toma de conciencia de lo evaluado.

\section{Segundo Taller: paneles de profesionales}

El segundo taller consistió en la realización de un panel de profesionales con el propósito de acercar las vivencias de profesionales egresados, en torno a las demandas actuales del contexto laboral (segundo eje) junto a la inclinación vocacional concretada. El panel estuvo conformado por docentes de nuestra casa de estudios, junto al Presidente del Colegio de Veterinarios-ex alumno de nuestra facultad de ciencias veterinarias, en una riqueza insondable, un testimonio de vida personal y profesional único, brindando claridad en los aspectos múltiples que la carrera de medicina veterinaria propicia. Se suma además a éste propósito la riqueza de la heterogeneidad constituida por los campos ocupacionales que cada cual desempeña y las funciones y roles que les atañen actualmente. La escucha y el intercambio ofrecieron un espacio de encuentro con lo laboral sumado a la experiencia subjetiva que socializaron y compartieron como referencias múltiples de la carrera y su formación. En la riqueza testimonial abordada destacaron el potencial asumido en las personas que conforman nuestra casa de estudio, de alto prestigio, quienes con total apertura y compromiso dijeron si al acompañamiento estudiantil.

\section{Tercer taller: entrevistas laborales}

El tercer taller abordó cuestiones claves para enfrentar una entrevista laboral y aspectos que permitieran una respuesta más ajustada a cada puesto de trabajo (tercer eje). La realización de la entrevista laboral en pos de construir competencias sociales para el abordaje de la misma, se concretó dando inicio con una dinámica de Role-Playing, donde en la asunción y adjudicación de roles, se reeditó la misma. La técnica del Role-Playing es una vía de formación y aprendizaje que tiene su aplicación en el área pedagógica de la formación experiencial y cognitiva construyendo un instrumento de intervención esencial para el desarrollo de habilidades (López Barbera y otros, 2000). Así el taller, se constituyó en el espacio para experimentar, un lugar para construir conocimiento personal y colectivo, donde se parte de lo que cada uno trae, que al ponerlo en juego con otras experiencias permite acceder a saberes más complejos. Este intercambio permitió mirarse en las prácticas, reconocerlas, reflexionarlas, tensionarlas y cuestionarlas. Observarse ellos mismos haciendo, siendo parte valiosa del conocimiento construido con otros y analizando cuáles han de ser las actitudes más adecuadas de respuesta ante el contexto laboral, resulto ser un desafío mayor. Luego de la realización de la dinámica se analizaron y detectaron ciertos errores frecuentes en el manejo de la entrevista vinculado a presencia de ansiedades, falta de organización, dificultades en la propia valoración e inseguridades.

\section{Cuarto taller: construcción del Curriculum Vitae}

El cuarto taller buscó brindar elementos y orientaciones específicas en la construcción del Curriculum Vitae (CV) y carta de presentación, como documentación necesaria para la solicitud de puestos laborales. Se estableció el análisis de los diferentes aspectos a tener en cuenta para la realización de un $\mathrm{CV}$, brindando orientaciones y tips para su facilitación y elaboración. Se modelaron algunos ejemplos para ir comparando los ya construidos con los nuevos por realizar, ejecutando los ajustes necesarios para responder a los criterios preestablecidos. Dentro de los aspectos que se han detallado para su construcción se referencian organización secuencial, tipo de letra utilizada, programas tecnológicos para su elaboración, uso de información relevante, normas generales, apartados, uso del lenguaje, experiencias concretadas e información complementaria entre otras.

\section{Resultados}

Teniendo en cuenta la realización de los cuatro talleres en torno a diversos ejes de trabajo, es que se comparten resultados organizados sobre la base de los mismos, con el fin de diferenciar la información para favorecer la lectura e interpretación. Se promovieron 
acciones que hicieran posible la indagación en las habilidades sociales que fueron construyendo los estudiantes, buscando tomar conciencia de cuáles resultan necesarias de abordar en un futuro laboral. En el proceso de interrogación aparecen factores que inciden notablemente en la percepción del mundo que se avecina, tomando como referencia algunas verbalizaciones entre las que mencionan falta de seguridad personal, desconocimiento de las propias capacidades, desvalorización, miedo a no ser competente, comodidad, no salir de la zona de confort y zona de aprendizaje restringida por evitar cambios o posibles errores. Para un mayor análisis se comparten resultados teniendo en cuenta cada uno de los talleres por separado a fin de acceder a un estudio pormenorizado de los mismos.

\section{Primer taller: autoconocimiento}

Considerando el primer taller - a partir del análisis de los resultados obtenidos luego de la implementación de la técnica EHS - se observa que una gran mayoría necesita potenciar las habilidades sociales, con escalas en declinación tales como autoexpresión en situaciones sociales (refleja la capacidad de expresarse uno mismo en forma espontánea, sin ansiedad, en distintas situaciones sociales: entrevistas laborales, en grupos y reuniones sociales), defensa de los propios derechos como consumidor (falta de autoconfianza), cortar interacciones (refleja la habilidad para cortar interacciones que no se quieren mantener, sea con un vendedor, con una conversación de un amigo o una relación con una persona con la que no se desea seguir saliendo, así como negarse a prestar algo cuando no se desea hacerlo) y hacer peticiones, todas ellas en valores descendidos. Considerando el valor de la temática los participantes-alumnos expresan en una gran mayoría que ha resultado ser pertinente e interesante, siendo la metodología de intervención dinámica en su totalidad, vinculando la experiencia con las emociones vividas, disfrutando de la propuesta y reflejando comodidad en la ejecución y participación. En los comentarios vertidos desde las experiencias personales se rescatan valoraciones que evidencian el interés por la temática, la detección de capacidades, fortalezas y debilidades, la necesidad de dinamizar los espacios de capacitación en torno al uso de una ade-cuada metodología y la utilidad observada en relación con el proceso de autoconocimiento alcanzado.

\section{Segundo taller: panel de profesionales}

Teniendo en cuenta el segundo taller en relación con la vivencia del panel se acercan las siguientes apreciaciones individuales registradas mediante encuestas de opiniones de características abiertas, que permiten dar cuenta del valor e importancia del mismo, a saber:

- Fue lo que necesitaba escuchar en este momento de mi vida.
- Es bueno saber esto y cómo afrontar las dificultades sabiendo que ya estoy por terminar.

- Muy bueno para saber qué hacer y cómo resolver conflictos.

- La enseñanza que rescato es: "Darle siempre para adelante".

- Me parece muy bueno que haya este tipo de charlas, resultó muy interesante y educativa aprender de las experiencias de otros veterinarios colegas.

El encuentro fue calificado como dinámico, interesante, disfrutable, cómodo, placentero. Las valoraciones vertidas enfatizan la necesidad y la satisfacción del acercamiento realizado en torno a la vinculación entre la teoría y la práctica, por estar en contacto directo con aquellas áreas ocupacionales que cada uno de los profesionales ha llevado a cabo. Esta instancia permitió configurar el futuro rol ocupacional y evaluar los riesgos, dificultades, necesidades que cada campo demanda en la preparación personal y social.

\section{Tercer taller: habilidades sociales y laborales}

El tercer taller relacionado con la concreción y realización de la entrevista laboral se pudieron observar en la dinámica del role-playing problemáticas evidentes en cuestiones relativas a la falta de seguridad personal, dificultad para trabajar en grupo, preocupaciones frecuentes sobre la falta de información brindada, falta de entrenamiento en habilidades sociales, necesidad de articular herramientas en el trabajo conjunto, compromisos en las valoraciones personales, apreciaciones desestimadas de sí mismos, falta de confianza, observación del entorno laboral como ciertamente complejo, atravesado por variables contextuales y con la presencia de cierta incertidumbre de lo que realizarán al salir del contexto académico.

\section{Cuarto taller: confección de Curriculum Vitae}

En el taller correspondiente al cuarto encuentro se trabajó acerca de la confección de un CV, se destacaron como resultados la importancia y valor que tiene el hecho de ampliar conocimientos permitiendo así un ajuste real del propio instrumento de presentación a un formato más interactivo y claro. Se realizaron ajustes que favorecieron su mayor adaptabilidad y desempeño con el fin de consolidar una herramienta de solicitud laboral más pertinente. Los estudiantes reconocen que ésta construcción no ha sido abordada con antelación y se requiere ir organizando la información con tópicos más relevantes.

\section{Conclusión}

Al observar el conjunto de valoraciones vertidas por los estudiantes desde cada una de las experiencias de los talleres se observa gusto, disfrute e interés por sostener estos espacios de formación, debido a que los mismos se constituyen en la antesala laboral y permiten brindar 
la posibilidad de atender a las necesidades futuras en un tiempo previo de preparación. Además, requieren continuidad y solicitan una mayor permanencia debido a que no sólo han configurado un espacio de encuentro consigo mismos y sus potencialidades, sino también con la realidad social y laboral.

En el ejercicio de la Medicina Veterinaria, se requiere interactuar y comunicarse de manera efectiva con los futuros clientes, ya que se encuentra en el núcleo de la actividad profesional y por lo tanto del bagaje competencial necesario para ejercerlas. Atribuir a la competencia social la capacidad para expresarse libremente, defender los derechos, lograr objetivos personales, maximizar las consecuencias positivas en los intercambios con interlocutores, minimizar la pérdida de reforzadores en el desempeño personal contribuye primordialmente a forjar personas que experimenten conductas deseables y de inserción personal, social y laboral adecuadas, permitiendo construir un rol profesional integral que fomente $y$ combine la integración interdisciplinaria para propiciar en los jóvenes un entrenamiento previo a la inmersión laboral. La universidad, como institución educadora debe tener dentro de sus prioridades otorgar y brindar alternativas y espacios formativos para los jóvenes, siendo que estas competencias permitan constituirse en una herramienta imprescindible para obtener éxito personal en todas las facetas de la vida, sabiendo que el ser humano gran parte de su tiempo se encuentra interactuando con otras personas, siendo su naturaleza social, resultando indispensable que se disponga de este repertorio de habilidades para que la comunicación y actividad profesional sean eficaces. Los espacios formativos se convierten en momentos e instancias necesarias para la formación integral, requiriendo abordajes preventivos que permitan ir forjando los cimientos esenciales a la hora del futuro egreso y consolidación de experiencias para fortificar el trabajo personal y grupal. Las experiencias que se vienen realizando desde el Servicio de Orientación Educativa se van nutriendo año a año con los aportes estudiantiles y docentes, sabiendo que la necesidad de su instrumentación es un pedido continuo que los mismos protagonistas realizan y que se requiere establecer un ordenamiento significativo para que estas cuestiones abordadas puedan tener continuidad. En las valoraciones de los cuatro años en los que se ha instaurado esta práctica se rescatan apreciaciones que dan cuenta del impacto vivenciado en las mismas y de las necesidades que se requieren abordar para que la formación técnica se complemente con lo humano y laboral. Por ello apostamos a que estos ofrecimientos puedan estar intervinculados con la estructura curricular para que su sostenimiento, abordaje e implementación no resulte ser sólo una instancia presentada desde el Servicio de Orientación Educativa sino una necesidad institucional detectada, para brindar las herramientas y caminos necesarios de construir en la elaboración de oportunidades únicas dadas desde la motivación estudiantil.

\section{Agradecimientos}

A todos los estudiantes que participaron de esta investigación. A los colaboradores de cada asignatura que forman parte de este proyecto. A los profesionales que participaron del segundo taller.

\section{Bibliografía}

Gismero González E. 2000. Escala de habilidades sociales. TEA Ediciones, Buenos Aires. 56 pp.

Le Boterf G. 2000. Ingeniería de las competencias. EPISE y Gestión 2000, Barcelona. 462 pp.

López Barbera E, Knappe, P. 2000. Introducción al role-playing pedagógico. Ed. Desclee de Brouwer, Madrid. 296 pp.

Perrenoud PH. 1997. Construire des compétences dès l'école. ESF Editeur. Paris. 125 pp.

Pozo M. 2008. Aprendices y maestros. La psicología cognitiva del aprendizaje. Editorial Alianza, Madrid. 614 pp.

Taylor SJ, Bogdan R. 2000. Introducción a los métodos cualitativos de investigación. Editorial Paidós, Barcelona. 345 pp.

Wagensberg J. 1998. Ideas para la imaginación impura. Tusquets Editores, Barcelona. 282 pp. 